\title{
Acute common peroneal nerve palsy caused by hemorrhage into a recurrent ganglion
}

\author{
EW Khairallah MD, MJ Weinberg MSc MD, J Mahoney MD FRCSC \\ Department of Plastic Surgery, Saint Michael's Hospital, Toronto, Ontario
}

Ganglia were first described by Hippocrates, as 'knots of tissue' containing 'mucoid flesh' (1). They occur most commonly at the wrist and occasionally at the knee. They usually present with pain and rarely cause compressive neuropathies.

Hartwell (2) in 1901 first described peripheral nerve (median nerve) involvement by a ganglion. In 1921, Sultan (3) reported a patient with paralysis of the common peroneal nerve caused by a cystic tumour. In 1952, Brooks (4) described 13 patients who had peripheral nerve compression by simple ganglia. In three of these patients the common peroneal nerve was involved. Stack et al in 1965 (5) described nine cases of compression of the common peroneal nerve by ganglia arising from around the knee. Following this series by Stack et al (5), there were no more cases of common peroneal nerve compression reported in the literature, until a report in 1994 by Evans et al (6). In this case magnetic resonance imaging was emphasized as a useful diagnostic modality.

We describe a case of acute common peroneal nerve compression neuropathy. The ganglion was recurrent following previous incomplete excisions. The acute compression was likely caused by hemorrhage into the ganglion. This case emphasizes the importance of early and complete surgical excision of ganglia around the fibula neck.

\section{CASE REPORT}

A 39-year-old male labourer was referred to our outpatient clinic with a fourweek history of progressive pain, foot drop and numbness over the dorsum of the foot. These symptoms all started suddenly and were aggravated by walking and at the end of the day. The pain was excruciating. The foot drop was marked and hindered ambulation. There was no history of trauma.

The patient first noticed a mass at the upper lateral leg 12 years earlier. The mass was surgically excised in 1977 and 1982. The pathology on each occasion was that of a ganglion. Each time the patient was told that the mass was not completely excised for fear of damage to the common peroneal nerve. The ganglion recurred seven to eight years after the second surgery (five years ago). The patient has had numerous aspirations following the recurrence.

Clinical examination revealed a mobile, nontender mass, $7 \times 8 \mathrm{~cm}$, over the neck of the right fibula. There was no movement in the extensor-hallucis longus (EHL:MRC 0/5). Dorsiflexion and eversion of the right foot was markedly reduced with a power grade of $4 / 5$. Sensation was diminished in the distribution of the superficial and deep peroneal nerves. Tinel's sign was negative.

Initial investigation with plain radiographs was normal except for a soft tissue swelling at the neck of the fibula (Figure 1). Ultrasonography confirmed the cystic nature 
of the mass (Figure 2). Computerized tomographic (CT) scan (Figure 3) showed a multiloculated, thin walled, low density mass around the neck of the fibula. The common peroneal nerve was not visualized.

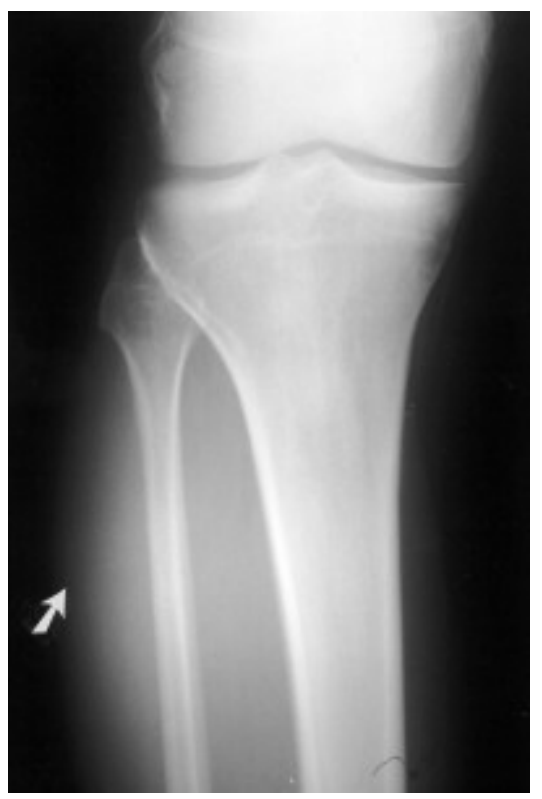

Figure 1) Plain $x$-ray of knee confirming soft tissue swelling (arrow)

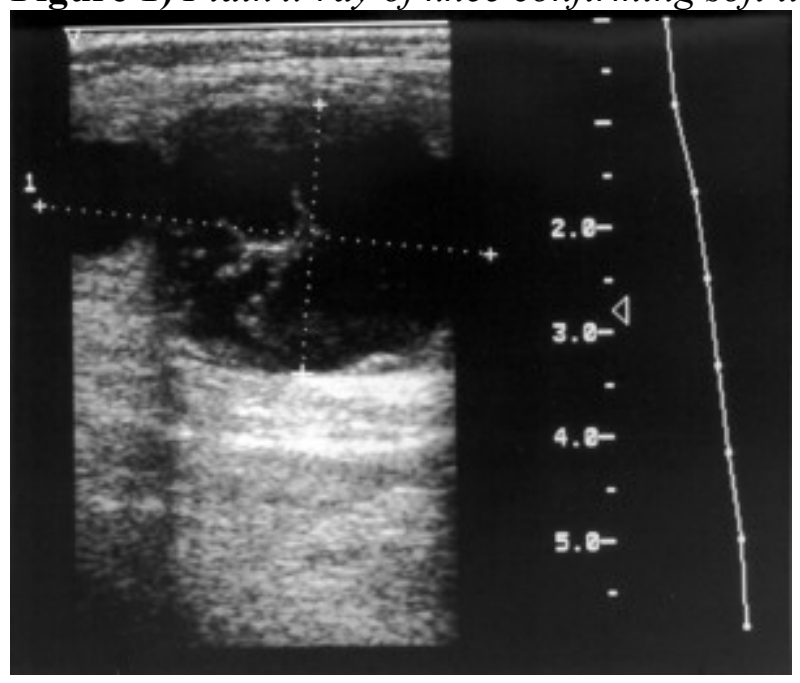

Figure 2) Ultrasound of lower leg showing multiloculated cystic mass

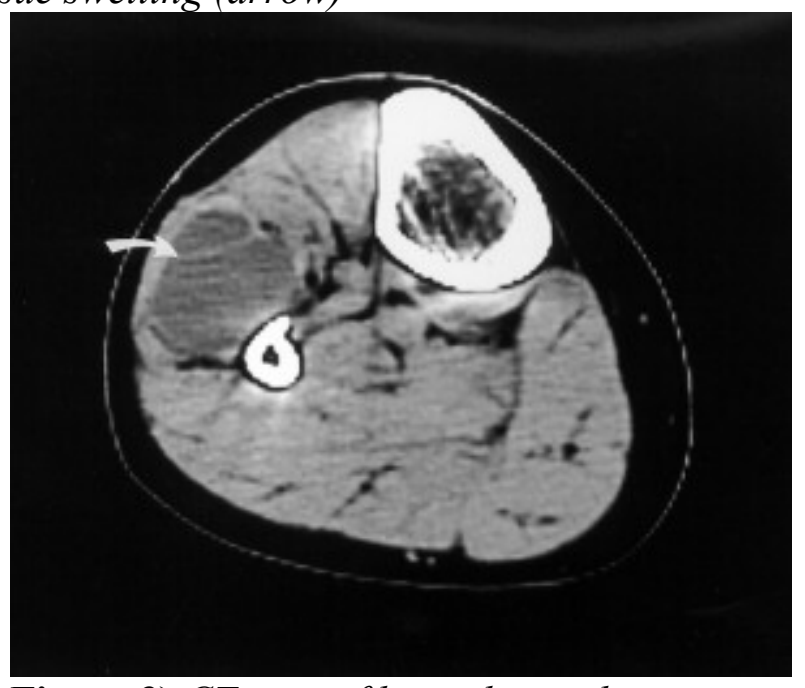

Figure 3) CT scan of lower leg in the region of the fibular head showing a multilocular cystic mass (white arrow) 
Surgical exploration was performed. There was a $3 \times 5 \mathrm{~cm}$ thin walled cystic mass deep to the deep fascia ( Figure 4, left) that was directly compressing the common peroneal nerve. The mass was found to arise from the tibiofibular joint (Figure 4, right). The mass and a cuff of joint capsule were excised. When the mass was opened the walls were blood stained and the cyst was filled with blood clot. The pathology report confirmed the presence of blood in the cavity and in the walls.
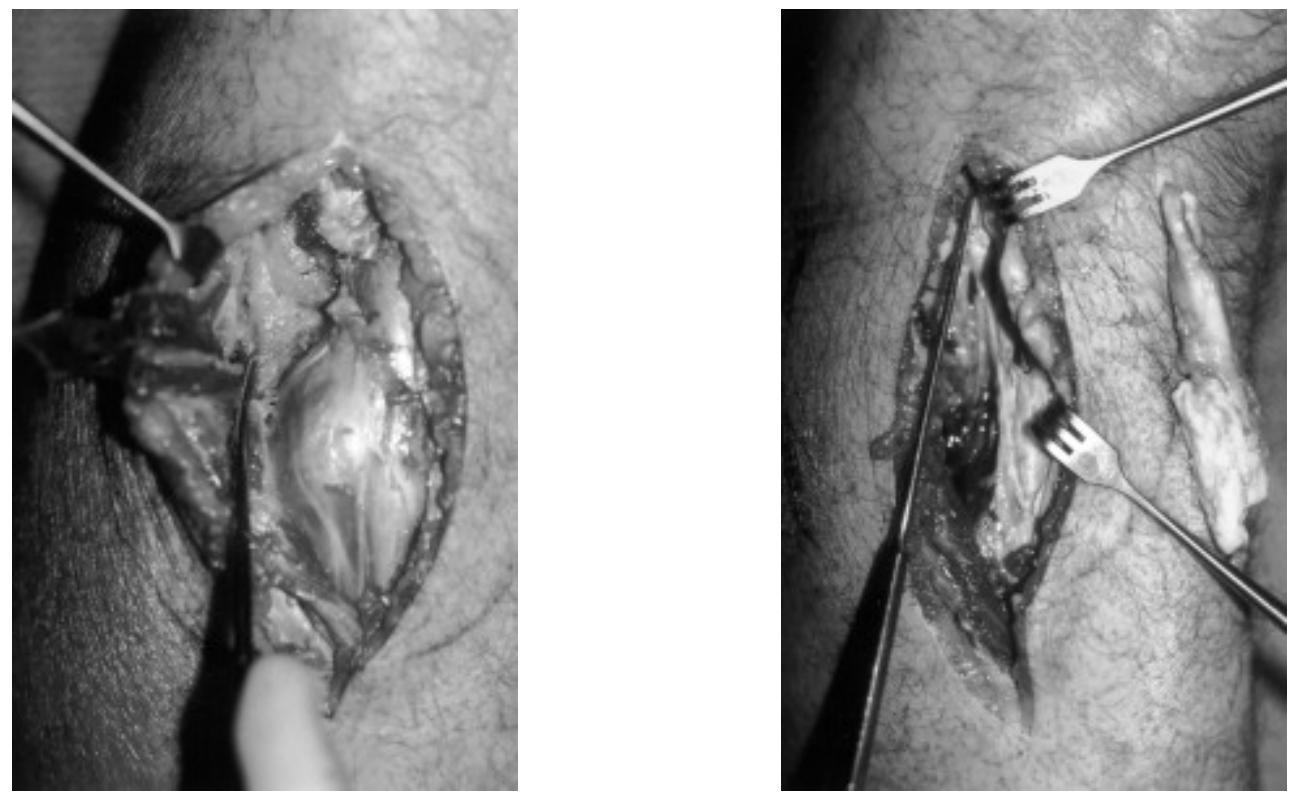

Figure 4) Intraoperative findings. Left. Ganglion compressing the common peroneal nerve (skin hook). Right. The pedicle of the ganglion arising from the tibiofibular joint (at the end of the skin hook)

At the six month follow-up there was no evidence of recurrence. The patient was pain free. Motor function was $4+/ 5$ for foot eversion and dorsiflexion and EHL power was $4 / 5$. Sensation was slightly diminished in the previously involved territories.

\section{DISCUSSION}

Ganglia usually present with pain and rarely with symptoms and signs secondary to a compressive neuropathy. If a compressive neuropathy exists the most common symptom is still pain that has a delayed onset, is mild to moderate in intensity and is in the distribution of the nerve. Less frequently the patients complain of weakness and of numbness and tingling. These symptoms can be aggravated by muscle use and ameliorated with rest. This suggests that dynamic factors in addition to compression of the nerve by ganglia are in effect (7). The interesting features of our case are the acute nature and severity of the symptoms. The sudden onset of symptoms and the operative finding of hemorrhage into the ganglion suggest this as a likely mechanism.

Radiological studies were useful in confirming the diagnosis and determining the extent of the lesion. Recent reports suggest magnetic resonance imaging (MRI) as a useful modality $(6,8)$. 
Treatment of ganglia is controversial. The advocates of surgery for wrist ganglia insist that high recurrence rates are related to incomplete excision of joint capsule (9). The fact that the ganglion in our patient recurred promptly after incomplete excision and was found to arise from the tibiofibular joint is consistent with this. We advocate early excision with removal of the joint capsule as the treatment of choice in the presence of a compression neuropathy. This is emphasized by the data of Stack et al (5), where the two patients that had poor recovery of motor function had foot drop for the longest time.

In summary, ganglia at the knee can cause significant neurological sequelae. The work-up should include an ultrasound and a CT scan. Surgical excision should be prompt and should include a cuff of tissue at the origin of the ganglion.

\section{REFERENCES}

1. Hipprocates: De Artucularis. Edition Anutio Foesio, 1657, p 806. An English translation by Witherington ET: Hippocrates, on Joints, London: William Heinemann, 1927;iii:277.

2. Hartwell AS. Cystic tumor of median nerve; Operation; Restoration of function. Boston Med Surg J 1901;144:582-3.

3. Sultan C. Ganglion der nervenscheide des nervus peroneus. Zentralbl F Chir 1921;48:963-5.

4. Brooks DM. Nerve compression by simple ganglia. A review of thirteen collected cases. J Bone Joint Surg 1952;34-B:391-400.

5. Stack RE, Bianco AJ, MacCarthy CS. Compression of the common peroneal nerve by ganglion cysts. J Bone Joint Surg 1965;47A:773- 8.

6. Evans ID, Neumann L, Frostick SP. Compression neuropathy of the common peroneal nerve caused by a ganglion. Microsurgery 1994;15:193-5.

7. Spinner A, Spencer PS. Nerve compression lesions of the upper extremity. A clinical and experimental review. Clin Orthop 1974;104:46-7.

8. Takagishi K, Maeda K, Ikeda T, Itoman M, Yamamoto M. Ganglion causing paralysis of the suprascapular nerve. Diagnosis by MRI and ultrasonography. Acta Orthopaedica Scandinavica 1991;62:391-3.

9. Angelides AC, Wallace PF. The dorsal ganglion of the wrist: Its pathogenesis, gross and microscopic anatomy, and surgical treatment. J Hand Surg 1976;1:228-35. 